\title{
Retention of Gadolinium-Based Contrast Agents in Multiple Sclerosis: Retrospective Analysis of an 18-Year Longitudinal Study
}

(D) Y. Forslin, DS. Shams, (D) F. Hashim, (D). Aspelin, (D) G. Bergendal, (D). Martola, DS. Fredrikson, (D) M. Kristoffersen-Wiberg, and (DT. Granberg

on $\equiv$

\begin{abstract}
BACKGROUND AND PURPOSE: Gadolinium-based contrast agents have been associated with lasting high T1-weighted signal intensity in the dentate nucleus and globus pallidus, with histopathologically confirmed gadolinium retention. We aimed to longitudinally investigate the relationship of multiple gadolinium-based contrast agent administrations to the Signal Intensity Index in the dentate nucleus and globus pallidus and any associations with cognitive function in multiple sclerosis.
\end{abstract}

MATERIALS AND METHODS: The Signal Intensity Index in the dentate nucleus and globus pallidus was retrospectively evaluated on T7-weighted MR imaging in an 18-year longitudinal cohort study of 23 patients with MS receiving multiple gadolinium-based contrast agent administrations and 23 healthy age- and sex-matched controls. Participants also underwent comprehensive neuropsychological testing.

RESULTS: Patients with MS had a higher Signal Intensity Index in the dentate nucleus $(P<.001)$, but not in the globus pallidus $(P=.19)$, compared with non-gadolinium-based contrast agent-exposed healthy controls by an unpaired $t$ test. Increasing numbers of gadoliniumbased contrast agent administrations were associated with an increased Signal Intensity Index in the dentate nucleus $(\beta=0.45, P<.001)$ and globus pallidus $(\beta=0.60, P<.001)$. This association remained stable with corrections for the age, disease duration, and physical disability for both the dentate nucleus $(\beta=0.43, P=.001)$ and globus pallidus $(\beta=0.58, P<.001)$. An increased Signal Intensity Index in the dentate nucleus among patients with MS was associated with lower verbal fluency scores, which remained significant after correction for several aspects of disease severity $(\beta=-0.40 P=.013)$.

CONCLUSIONS: Our data corroborate previous reports of lasting gadolinium retention in brain tissues. An increased Signal Intensity Index in the dentate nucleus and globus pallidus was associated with lower verbal fluency, which does not prove causality but encourages further studies on cognition and gadolinium-based contrast agent administration.

ABBREVIATIONS: DN = dentate nucleus; EDSS = Expanded Disability Status Scale; GBCA = gadolinium-based contrast agent; GP = globus pallidus; SII = Signal Intensity Index

G adolinium-based contrast agents (GBCAs) are an important aid in MR imaging diagnostics for improving the detection and characterization of pathologic processes. Approximately 30 million contrast-enhanced MRIs are performed every year, with an estimated 300 million administered thus far. ${ }^{1}$ In multiple scle-

Received October 22, 2016; accepted after revision March 3, 2017.

From the Departments of Clinical Science Intervention and Technology (Y.F., S.S., F.H., P.A., G.B., J.M., M.K.-W., T.G.), Radiology (Y.F., S.S., F.H., P.A., G.B., J.M., M.K.-W., T.G.), Neurology (G.B., S.F.), and Clinical Neuroscience (S.F.), Karolinska Institutet, Stockholm, Sweden.

This research was funded by the Stockholm County Council and Karolinska Institutet through the regional agreement on medical training and clinical research.

Please address correspondence to Yngve Forslin, MD, Department of Clinical Science, Intervention and Technology, Cl-46, Karolinska University Hospital, 14186 Stockholm, Sweden; e-mail: yngve.forslin@ki.se

-- Indicates open access to non-subscribers at www.ajnr.org

三 Indicates article with supplemental on-line table.

http://dx.doi.org/10.3174/ajnr.A5211

rosis, GBCAs play a key role in detecting disease activity. Gadolinium has paramagnetic properties that shorten the $\mathrm{T} 1$ relaxation in tissues, resulting in an increased signal on T1-weighted imaging. Because gadolinium $\left(\mathrm{Gd}^{3+}\right)$ is toxic, it is chelated to stabilize the compound and mitigate these effects. ${ }^{2}$ Macrocyclic GBCAs are kinetically more stable than linear GBCAs with less dissociation of gadolinium, while nonionic linear GBCAs are the least stable. ${ }^{3}$ Dissociation of gadolinium has been implicated in the development of nephrogenic systemic fibrosis, which is typically seen in patients with impaired renal function. ${ }^{2}$ This implication has led to the recommendation to primarily use macrocyclic GBCAs in patients with renal impairment. ${ }^{4}$

We know from earlier studies that gadolinium is deposited in the bone, liver, ${ }^{5,6}$ and skin. ${ }^{7}$ In 2014 , Kanda et $\mathrm{al}^{8}$ showed an association between the number of administrations of linear GBCA and a higher T1 intensity in the dentate nucleus (DN) and globus pallidus (GP), suggestive of gadolinium retention in the 


\begin{tabular}{|c|c|c|c|c|}
\hline & $\begin{array}{c}\text { Patients with MS, } \\
\text { Baseline }\end{array}$ & $\begin{array}{l}\text { Patients with MS, } \\
\text { 9-Year Follow-Up }\end{array}$ & $\begin{array}{l}\text { Patients with MS, } \\
\text { 18-Year Follow-Up }\end{array}$ & $\begin{array}{l}\text { Healthy } \\
\text { Controls }\end{array}$ \\
\hline Female/male (No.) & 18:5 & 18:5 & 18:5 & 18:5 \\
\hline Age (yr) & $39 \pm 8.1$ & $48 \pm 8.1$ & $57 \pm 8.0$ & $57 \pm 7.2$ \\
\hline Disease duration (yr) & $10 \pm 7$ & $19 \pm 7$ & $27 \pm 7$ & - \\
\hline MS subtype: RR/SP/PP (No.) & $18 / 5 / 0$ & $13 / 10 / 0$ & $3 / 20 / 0$ & - \\
\hline Disease-modifying therapy (No.) (\%) & $13(57 \%)$ & $13(57 \%)$ & $5(22 \%)$ & - \\
\hline Gadolinium administrations (median) (range) & $1(0-3)$ & $3(2-10)$ & $6(3-12)$ & 0 \\
\hline Dentate nucleus SII, middle cerebellar peduncle as a reference & $1.01 \pm 0.02$ & $1.03 \pm 0.03$ & $1.06 \pm 0.05$ & $1.01 \pm 0.03^{b}$ \\
\hline Globus pallidus SII, thalamus as a reference & $1.04 \pm 0.05$ & $1.06 \pm 0.04$ & $1.09 \pm 0.06$ & $1.10 \pm 0.04^{c}$ \\
\hline
\end{tabular}

Note:-PP indicates primary-progressive; RR, relapsing-remitting; SP, secondary-progressive.

${ }^{a}$ Values are reported as means, unless otherwise specified.

${ }^{\mathrm{b}}$ The difference in Slls between controls and patients with MS: unpaired $t$ test $(P<.001)$.

c The difference in Slls between controls and patients with MS: unpaired $t$ test $(P=.19)$.

brain. This finding has since been confirmed by other human studies, ${ }^{9-11}$ animal studies, and histopathologically. ${ }^{10,12-14}$ Concerns have therefore been raised regarding the safety profile of GBCAs and whether retention in brain tissue may have any longterm negative neurologic side effects. ${ }^{15}$ Recently, a large study in individuals with contrast-enhanced nonbrain MR imaging without a history of Parkinsonism failed to show any associations between administration of GBCA and Parkinsonism, but studies on cognitive aspects are lacking. ${ }^{16}$

We aimed to retrospectively investigate the association between the number of GBCA administrations and a higher Signal Intensity Index (SII) in the DN and GP in a longitudinal MS cohort with an 18-year follow-up and to compare these results with non-GBCA-exposed matched healthy controls. We also explored possible associations between a higher SII and cognitive disability in MS.

\section{MATERIALS AND METHODS Study Population}

On the basis of a consecutively recruited prospective cohort of 23 patients with MS followed for 18 years, we performed a retrospective analysis with regard to the SII. The cohort was originally recruited from the Department of Neurology in Huddinge, Karolinska University Hospital. The inclusion criterion at baseline was a diagnosis of MS according to the concurrent diagnostic criteria, ${ }^{17}$ while the exclusion criterion was neurologic comorbidities or MR imaging contraindications. For the study duration, all patients had at least 3 brain MR imaging scans. For the last MR imaging follow-up, we also recruited 23 age- and sex-matched healthy controls who were volunteers without neurologic diseases who were scanned with the same MR imaging protocol for the research project. The demography of the study population is presented in the Table. None of the participants had any history of renal or liver failure, and when available, renal and liver function blood test results were normal. The study was approved by the ethics review board in Stockholm, Sweden, and written informed consent was obtained from all participants.

\section{GBCA Administrations}

All patients with MS in the cohort received both linear nonionic gadodiamide (Omniscan; GE Healthcare, Piscataway, New Jersey) and linear ionic gadopentetate dimeglumine (Magnevist; Bayer HealthCare Pharmaceuticals, Wayne, New Jersey). Six patients had received macrocyclic gadoterate me- glumine (Dotarem; Guerbet, Aulnay-sous-Bois, France) on 1 occasion in the last 2 years before the last follow-up. The information on the number of administrations and class of GBCA was based on documentation in the digital radiologic information system, along with patient charts, to determine that no additional contrast-enhanced MR imaging had been performed at other sites.

\section{Imaging and Volumetry}

All patients underwent MR imaging of the brain at baseline, after 9 years, and after 18 years. MR imaging scans obtained between these time points were also included. MR imaging examinations were acquired with native 5-mm-thick T1-weighted spin-echo sequences at up to 3 time points (at least at baseline and 18-year follow-up). MPRAGE sequences (resolution, $1 \times 1 \times 1.5 \mathrm{~mm}$ ) were available at up to 4 time points during the last 9 years of the study (at least at 9- and 18-year follow-ups). The On-line Table details the MR imaging acquisition parameters.

The longitudinal stream of FreeSurfer 5.3.0 (http://surfer. nmr.mgh.harvard.edu) was used to obtain brain parenchymal fraction measurements from the MPRAGE scans with semimanual correction of topologic errors. ${ }^{18}$ Lesion segmentations were performed by using the combination of MPRAGE and FLAIR scans with the lesion growth algorithm in the Segmentation Toolbox 1.2.3 (Technische Universität, München, Munich, Germany) for SPM8 software (http://www.fil.ion.ucl. ac.uk/spm/software/spm12). ${ }^{19}$

\section{Radiologic Evaluation}

The SII was calculated according to the following formula: SII = Signal Intensity ROI $_{\text {Signal Intensity }}$ Reference Region. The radiologic measurements were performed by placing ROIs bilaterally in the GP and DN, with the thalamus and left middle cerebellar peduncle measured in the same image section as the reference points, respectively. The radiologic evaluation was blinded to the examination date, the original radiologic reading, and all clinical information. The specific region for each measurement was decided by consensus, by a radiology resident (Y.F.) and a neuroradiologist (F.H.), with simultaneous assessment of T2weighted images to improve the delineation of the $\mathrm{DN}$ and avoid placement of ROIs in lesioned areas. In 7 scans, measurements were not performed in the GP due to lesions in the ROIs and/or in the reference regions. 


\section{Clinical Data}

Patients were neurologically and neuropsychologically evaluated at 3 time points during the study (baseline, 9-year follow-up, and 18-year follow-up). Physical disability was assessed by a senior consultant in neurology (S.F.) with the Expanded Disability Status Scale (EDSS). ${ }^{20}$ The patients with MS underwent extensive neuropsychological testing performed by a senior neuropsychologist (G.B.), including the following: the Symbol Digit Modalities Test to assess information-processing speed; the F-A-S Test for evaluating phonologic verbal fluency; the Rey-Osterrieth Complex Figure Test-Copy for evaluating visuospatial ability; and the Rey Auditory Verbal Learning Test with encoding and delayed recall at 30 minutes for episodic auditory-verbal memory. The raw test scores were converted to normalized $z$-scores on the basis of age, sex, and educational level. ${ }^{21}$

\section{Statistical Analysis}

Descriptive statistics are presented as means and SDs. The SIIs of the right and left DN and GP were analyzed as individual data points because they were not always available in a paired fashion because the lesioned areas were not measured. The SII values for the GP and the cognitive $z$-scores for the Rey Auditory Verbal Learning Test and Rey-Osterrieth Complex Figure Test-Copy were negatively skewed and therefore underwent a reflect and logarithmic transformation [Lg10(Largest Score in Data +1) Data] to reach normal distribution. An unpaired $t$ test was used to compare the SII between controls and patients with MS obtained in the same scanner.

Multiple linear regression analyses were performed in only the patients with MS. They were used to evaluate associations between SIIs in the GP/DN (dependent variables) and the total number of both linear and all types of GBCA administrations (independent variable), corrected for MR imaging scanner, sequence, and age. Additional analyses corrected for MS disease severity were performed to avoid MS disease progression as a possible confounder. In a first step, disease duration and EDSS scores were added to the model. Second, the model was also corrected for lesion volume and brain parenchymal fraction (when volumetric data were available during the later 9 years of the study). Repeated-measures analysis of variance on the increasing number of GBCA administrations (stratified as $0,1-4$, and $>5$ administrations) was performed to assess longitudinal change. Associations with cognition were similarly investigated with multiple linear regression analysis between cognitive $z$-scores (dependent variable) and the SII (independent variable) with the same correction steps. All regression coefficients are presented as the standardized coefficient, $\beta$. SPSS (Version 22.0 for Mac; IBM, Armonk, New York) was used for statistical analysis. An $\alpha$ level of .05 was considered statistically significant, equaling a corrected level of .029 after adjustments for the false discovery rate according to the Benjamini-Hochberg procedure. $^{22}$

\section{RESULTS}

\section{GBCA Administration and Signal Intensity}

As detailed in the Table, the SII in the DN was higher in patients with MS than in healthy controls $(P<.001$, by the unpaired $t$
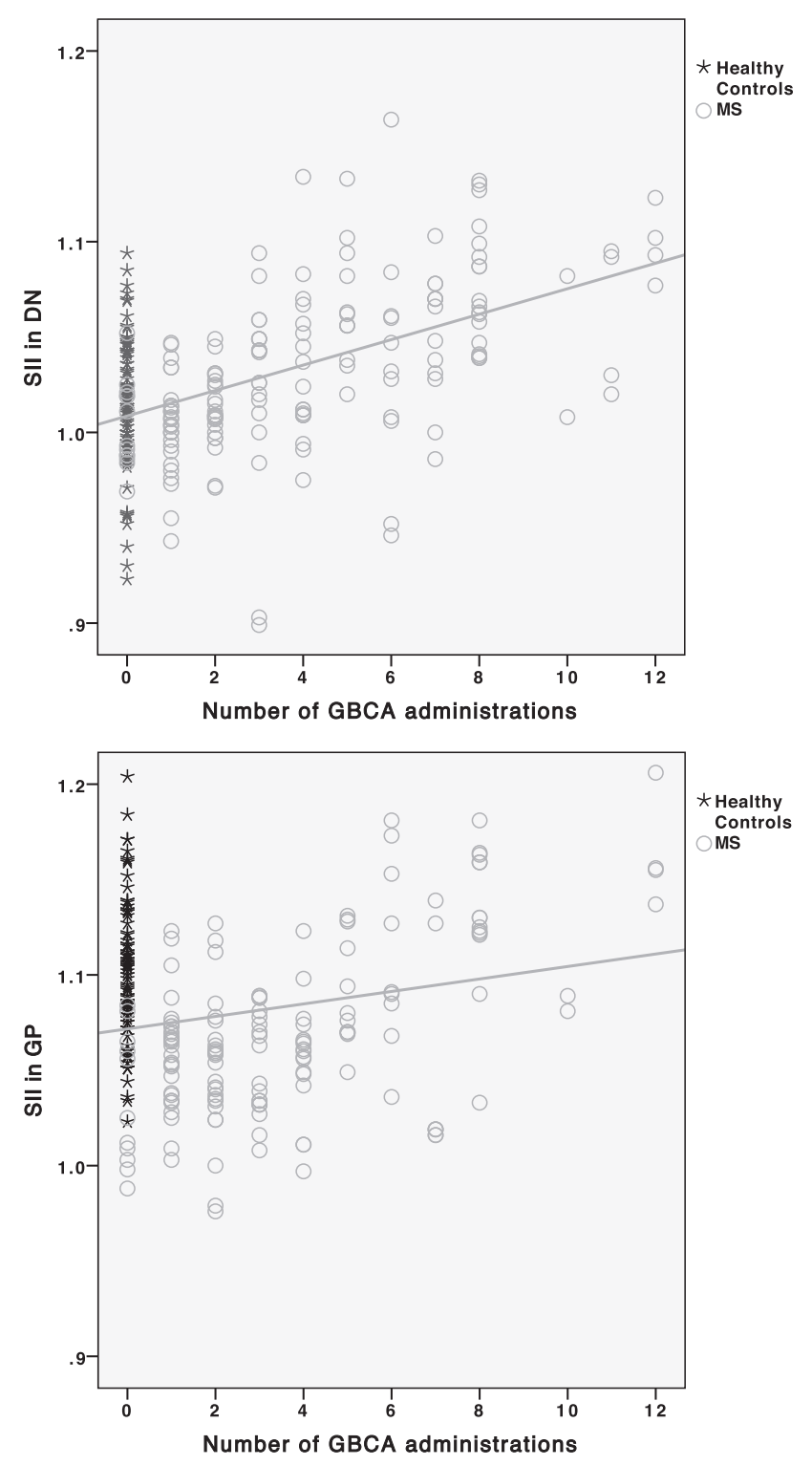

FIG 1. Scatterplots with linear regression lines showing that the number of GBCA administrations is associated with a higher SII in the DN and $\mathrm{GP}(\beta=0.45, P<.001$ and $\beta=0.60, P<.001$, respectively) with corrections for age, scanner, and sequence type.

test), while there was no significant difference for the GP ( $P=$ .19). Within the MS group, an increased number of GBCA administrations was associated with increased SIIs in both the DN $(\beta=0.45, P<.001)$ and $\mathrm{GP}(\beta=0.60, P<.001)$, illustrated in Fig 1. The associations between high SIIs in the $\mathrm{DN}(\beta=0.45, P<$ $.001)$ and GP $(\beta=0.58, P<.001)$ remained significant when using only the number of linear GBCAs as an independent variable. When adding corrections for MS disease duration and EDSS, a higher number of GBCA administrations was still associated with higher SIIs in the $\mathrm{DN}(\beta=0.43, P<.001)$ and $\mathrm{GP}(\beta=0.58$, $P<.001)$. When we analyzed the later time points when lesion volume and brain parenchymal fraction were available (as a further characterization of disease severity) and by adding correction for those factors in the regression model, a higher number of GBCA administrations remained associated with higher SIIs in the $\mathrm{DN}(\beta=0.39, P=.007)$ and GP $(\beta=0.64, P<$ 


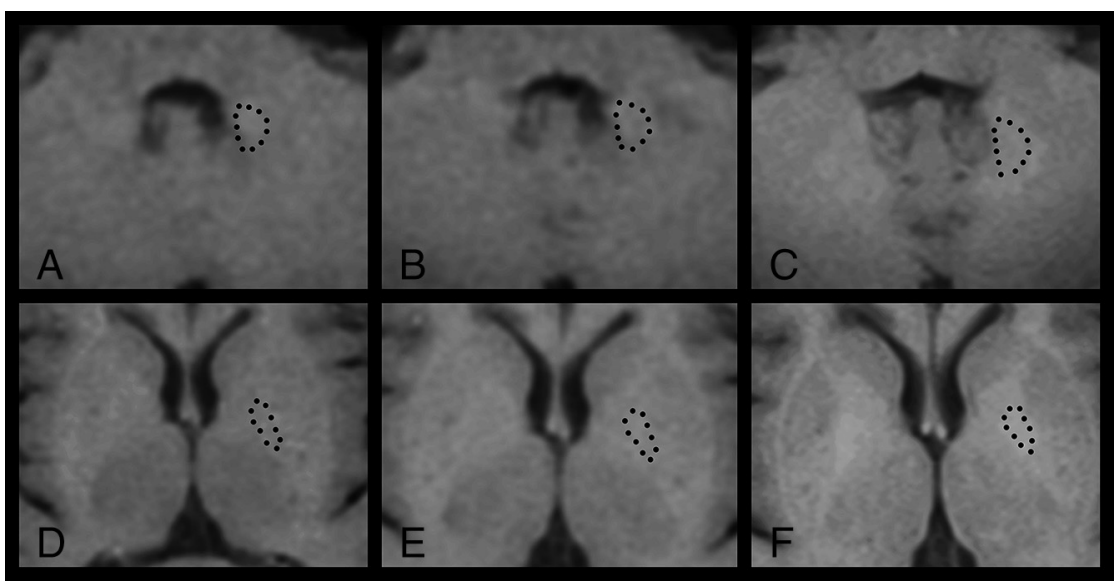

FIG 2. Longitudinal T1-weighted spin-echo signal-intensity changes in the $D N(A-C)$ and $G P(D-F)$ in a female patient with MS after receiving $0(A$ and $D), 1(B$ and $E$ ), and 8 ( $C$ and $F)$ GBCA administrations. This patient had an increasingly higher SII in both regions: DN $1.03(A), 1.05(B), 1.09$ $(C)$, GP $1.00(D), 1.12(E), 1.16(F)$. Dotted lines illustrate the outlining of the ROls. the $\mathrm{DN}(\beta=-0.40, P=.013)$, with a similar trend in the GP $(\beta=-0.36, P=$ $.034)$.

\section{DISCUSSION}

In the current study, we show that an increasing number of GBCA administrations is longitudinally associated with a higher SII in both the DN and GP in patients with MS, adding to the growing body of evidence of gadolinium retention in the brain. We further show some initial tentative results regarding associations of the SII in these structures with cognitive performance, which remained significant for verbal fluency when correcting for MS disease severity.

Investigations on gadolinium retention in the brain have studied different types of GBCAs. Multiple studies have .001). Figure 2 illustrates the longitudinal increase in DN signal intensity in relation to more GBCA administrations in 1 patient with MS.

From a longitudinal analysis perspective, the average SII change between baseline and the 18-year follow-up in patients was 0.06 in the DN and 0.07 in the GP, equaling a change of 0.009 in both the DN and GP per additional GBCA administration. Repeated-measures ANOVA (with the GreenhouseGeisser correction) showed that the SIIs were significantly different with respect to the number of administrations for the $\mathrm{DN}[F(2)=22.98, P<.001]$ and the GP $[F(1.07,10.67=6.29)$, $P=.028]$. Pair-wise comparisons were significant between 0 and 5-12 administrations for both the DN (1.01 \pm 0.24 and $1.08 \pm 0.032, P<.001)$ and the GP $(0.96 \pm 0.128$ and $1.09 \pm$ $0.53, P=.013)$. Similar trends were seen in the comparison between 0 and $1-4$ administrations (DN, $P=.049$; GP, $P=$ $.064)$.

\section{Associations with Cognition in Patients with MS}

A high SII in the GP $(\beta=-0.45, P<.001)$ was associated with low verbal fluency performance, and a similar trend was seen for the $\mathrm{DN}(\beta=-0.25, P=.03)$. After we corrected for disease duration and the EDSS, the associations were significant in both the DN $(\beta=-0.28, P=.012)$ and GP $(\beta=-0.49, P<.001)$. A higher SII in the GP $(\beta=-0.35, P=.003)$, but not in the $\mathrm{DN}$, was associated with low auditory verbal learning encoding $(\beta=$ $-0.35, P=.003)$ and low auditory verbal learning retention $(\beta=$ $-0.29, P=.01)$. These associations remained significant when correcting for disease duration and EDSS (encoding, $\beta=-0.33$, $P=.006$; retention, $\beta=-0.34, P=.004$ ). There were no significant associations between the SII in the DN or GP with either the Rey-Osterrieth Complex Figure Test or the Symbol Digit Modalities Test. After we corrected for disease duration and EDSS, a higher SII in the GP tended to be associated with a lower Symbol Digit Modalities Test score $(\beta=-0.24, P=.043)$. When we analyzed the later time points and added correction for lesion volume and brain parenchymal fraction, only the association between a higher SII and low verbal fluency remained significant in demonstrated associations between the SII in the DN and the number of linear GBCA administrations. ${ }^{23}$ More recently, a similar initial association was reported for macrocyclic GBCAs, ${ }^{24}$ and small amounts of retained gadolinium were recently found in human brain tissue after macrocyclic GBCA. ${ }^{13}$ However, more recent imaging studies regarding gadolinium retention support the use of macrocyclic GBCA as a safer choice even for patients with normal renal function. ${ }^{23,25,26}$ In our study, all patients received linear GBCAs, and 6 of 23 patients also received 1 administration of a macrocyclic GBCA. However, when we studied the patients with MS who received a linear GBCA (gadodiamide and gadopentetic acid) solely, the results were relatively unchanged.

Some studies have used the pons as a reference region for the $\mathrm{DN}$, but here we chose the left middle cerebellar peduncle instead because it was easier to find nonlesioned areas for reference that were not affected by phase-dependent artifacts on T1-weighted spin-echo scans. ${ }^{9,11,25}$ In line with previous studies, the thalamus was used as a reference point for the GP with special care to avoid lesions, though we note that diffuse MS disease-related changes in the thalamus may be a possible confounder. ${ }^{27}$

We have tried to carefully mitigate the confounding effects of MS pathology or progression in our analysis. However, MS typically causes hypointensities in both white and gray matter on T1weighted imaging, ${ }^{28}$ which would likely cause an underestimation of the SII rather than an overestimation in the ROI. On the other hand, we note that a hyperintense $\mathrm{DN}$ on native T1-weighted MR imaging has previously been reported in secondary-progressive MS. ${ }^{29}$ Whether these previous results have been confounded by GBCA administrations (before current knowledge) or actually reflect disease-related signal changes remains unclear. The different scanners and sequences used are a limitation but also unavoidable when performing a long-term study with such long-term follow-up because there is a clinical need for scanner upgrades with time. Because these patients were part of a prospective MS study, they had been scanned with the follow-up similar to that of MR imaging protocols for T1 spin-echo and MPRAGE, respectively, but on different scanners during the years. A subgroup analysis in 
a previous study did not show any significant difference when using different sequences. ${ }^{23}$ However, a later study showed that this significantly influenced the results, highlighting the importance of using the same sequences and scanners if possible. ${ }^{30,31}$ On the basis of these previous results, we therefore corrected the measurements for these factors to mitigate any scanner-related effects.

In the current study, we showed that a higher SII in the DN in patients with MS is associated with lower word fluency scores, which was the only cognitive measurement that remained associated with the SII after correction for several aspects of MS severity. Most interesting, executive function, including verbal fluency, is less frequently affected compared with episodic memory and information-processing speed in MS. ${ }^{32}$ Verbal fluency also declines after pallidotomy and cerebellar damage; this finding highlights the importance of these structures in verbal fluency. ${ }^{33,34}$ Furthermore, a recent study in MS showed that higher functional connectivity between the DN and prefrontal, motor, and parietal cortices is associated with lower structural parenchymal damage and less clinical impairment. ${ }^{35}$ However, many other areas of the brain are affected by MS, which may be more closely related to the decline in our neuropsychological test results. ${ }^{32}$ Naturally, it is of great clinical interest to explore any possible negative neurologic or cognitive adverse effects of gadolinium retention. It is, however, important to stress that our retrospective results must be interpreted with caution and do not necessarily imply causation.

It is not clear to what degree factors such as renal failure, liver dysfunction, and genetic variations might influence the risk of developing a higher SII in the brain after repeated GBCA administrations. MS is one of the largest patient groups receiving repeat GBCA administrations and may, therefore, be at special risk of accumulation in the brain. ${ }^{36} \mathrm{MS}$ also has a negative impact on cognitive function. ${ }^{32}$ Consequently, it is difficult to separate the effects of MS progression and a hypothetic effect on cognition attributed to GBCA, though we statistically corrected for the potential impact of disease severity. Furthermore, disease duration, lesion volume, and brain parenchymal fraction might not fully adjust for an MS-dependent decline of brain function because MS is a heterogeneous disease with individualized treatment regimens, different progression rates, and cognitive reserve, ${ }^{37}$ which are not completely represented by these biomarkers. Last, another limitation was the lack of a matched MS group not exposed to GBCAs or solely to macrocyclic GBCAs, because it was not possible to find a large-enough group of such patients with a disease duration similar to that of the studied cohort.

The strengths of the study are the long follow-up with available clinical, laboratory, and radiologic information and a study population representative of the MS panorama with different subtypes, disease duration, and EDSS scores.

\section{CONCLUSIONS}

Our data add support to the existing framework that gadolinium may have lasting effects on the T1-weighted intensities in the DN and GP. Although we show that a higher SII is associated with worse verbal fluency scores, these results are only exploratory and have to be interpreted with caution. Future studies are important to further investigate these cognitive aspects and other clinical outcome variables in other cohorts with a repeat number of GBCA administrations to understand their clinical implications.

\section{ACKNOWLEDGMENTS}

We thank the study participants for making this study possible.

Disclosures: Yngve Forslin-RELATED: Grant: ALF, Comments: This research was funded by Stockholm County Council and Karolinska Institutet through the regional agreement on medical training and clinical research (ALF grant)*. Farouk HashimRELATED: Grant: ALF*. Gösta Bergendal—RELATED: Grant: ALF*. Sten FredriksonUNRELATED: Board Membership: Chairman of the Educational and Examination Committee of the Swedish Neurological Society and Swedish delegate of the EFNS, European Board of Neurology and MSIF International Medical and Scientific Board; OTHER RELATIONSHIPS: received honorarium for lectures, educational activities, and/or consultancy from Genzyme/Sanof, Merck, Novartis, and Teva Pharmaceutical Industries. Tobias Granberg-RELATED: Grant: Stockholm City Council* *Money paid to the institution.

\section{REFERENCES}

1. Lohrke J, Frenzel T, Endrikat J, et al. 25 years of contrast-enhanced MRI: developments, current challenges and future perspectives. Adv Ther 2016;33:1-28 CrossRef Medline

2. Idée JM, Fretellier N, Robic C, et al. The role of gadolinium chelates in the mechanism of nephrogenic systemic fibrosis: a critical update. Crit Rev Toxicol 2014;44:895-913 CrossRef Medline

3. Frenzel T, Lengsfeld P, Schirmer H, et al. Stability of gadoliniumbased magnetic resonance imaging contrast agents in human serum at 37 degrees C. Invest Radiol 2008;43:817-28 CrossRef Medline

4. Thomsen HS, Morcos SK, Almén T, et al; ESUR Contrast Medium Safety Committee. Nephrogenic systemic fibrosis and gadoliniumbased contrast media: updated ESUR Contrast Medium Safety Committee guidelines. Eur Radiol 2013;23:307-18 CrossRef Medline

5. White GW, Gibby WA, Tweedle MF. Comparison of Gd(DTPABMA) (Omniscan) versus Gd(HP-DO3A) (ProHance) relative to gadolinium retention in human bone tissue by inductively coupled plasma mass spectroscopy. Invest Radiol 2006;41:272-78 CrossRef Medline

6. Idée JM, Port M, Dencausse A, et al. Involvement of gadolinium chelates in the mechanism of nephrogenic systemic fibrosis: an update. Radiol Clin North Am 2009;47:855-69, vii CrossRef Medline

7. Roberts DR, Lindhorst SM, Welsh CT, et al. High levels of gadolinium deposition in the skin of a patient with normal renal function. Invest Radiol 2016;51:280-89 CrossRef Medline

8. Kanda $\mathrm{T}$, Ishii $\mathrm{K}$, Kawaguchi $\mathrm{H}$, et al. High signal intensity in the dentate nucleus and globus pallidus on unenhanced T1weighted MR images: relationship with increasing cumulative dose of a gadolinium-based contrast material. Radiology 2014;270: 834-41 CrossRef Medline

9. Errante Y, Cirimele V, Mallio CA, et al. Progressive increase of T1 signal intensity of the dentate nucleus on unenhanced magnetic resonance images is associated with cumulative doses of intravenously administered gadodiamide in patients with normal renal function, suggesting dechelation. Invest Radiol 2014;49:685-90 CrossRef Medline

10. McDonald RJ, McDonald JS, Kallmes DF, et al. Intracranial gadolinium deposition after contrast-enhanced MR imaging. Radiology 2015;275:772-82 CrossRef Medline

11. Quattrocchi CC, Mallio CA, Errante Y, et al. Gadodiamide and dentate nucleus $\mathrm{T} 1$ hyperintensity in patients with meningioma evaluated by multiple follow-up contrast-enhanced magnetic resonance examinations with no systemic interval therapy. Invest Radiol 2015; 50:470-72 CrossRef Medline

12. Kanda T, Fukusato T, Matsuda M, et al. Gadolinium-based contrast agent accumulates in the brain even in subjects without severe renal dysfunction: evaluation of autopsy brain specimens with inductively coupled plasma mass spectroscopy. Radiology 2015;276: 228-32 CrossRef Medline

13. Murata N, Gonzalez-Cuyar LF, Murata K, et al. Macrocyclic and 
other non-group 1 gadolinium contrast agents deposit low levels of gadolinium in brain and bone tissue: preliminary results from 9 patients with normal renal function. Invest Radiol 2016;51:447-53 CrossRef Medline

14. Robert P, Lehericy S, Grand S, et al. T1-weighted hypersignal in the deep cerebellar nuclei after repeated administrations of gadolinium-based contrast agents in healthy rats: difference between linear and macrocyclic agents. Invest Radiol 2015;50:473-80 CrossRef Medline

15. Goischke HK. MRI with gadolinium-based contrast agents: practical help to ensure patient safety. J Am Coll Radiol 2016;13:890 CrossRef Medline

16. Welk B, McArthur E, Morrow SA, et al. Association between gadolinium contrast exposure and the risk of parkinsonism. JAMA 2016; 316:96-98 CrossRef Medline

17. Poser CM, Paty DW, Scheinberg L, et al. New diagnostic criteria for multiple sclerosis: guidelines for research protocols. Ann Neurol 1983;13:227-31 CrossRef Medline

18. Reuter M, Schmansky NJ, Rosas HD, et al. Within-subject template estimation for unbiased longitudinal image analysis. Neuroimage 2012;61:1402-18 CrossRef Medline

19. Schmidt P, Gaser C, Arsic M, et al. An automated tool for detection of FLAIR-hyperintense white-matter lesions in multiple sclerosis. Neuroimage 2012;59:3774-83 CrossRef Medline

20. Kurtzke JF. Rating neurologic impairment in multiple sclerosis: an expanded disability status scale (EDSS). Neurology 1983;33:1444-52 CrossRef Medline

21. Lezak MD, ed. Neuropsychological Assessment. 5th ed. Oxford: Oxford University Press; 2012

22. Benjamini Y, Krieger AM, Yekutieli D. Adaptive linear step-up procedures that control the false discovery rate. Biometrika 2006;93: 491-507 CrossRef

23. Radbruch A, Weberling LD, Kieslich PJ, et al. Gadolinium retention in the dentate nucleus and globus pallidus is dependent on the class of contrast agent. Radiology 2015;275:783-91 CrossRef Medline

24. Stojanov DA, Aracki-Trenkic A, Vojinovic S, et al. Increasing signal intensity within the dentate nucleus and globus pallidus on unenhanced T1W magnetic resonance images in patients with relapsingremitting multiple sclerosis: correlation with cumulative dose of a macrocyclic gadolinium-based contrast agent, gadobutrol. Eur Radiol 2016;26:807-15 CrossRef Medline

25. Kanda $\mathrm{T}$, Osawa $\mathrm{M}$, Oba $\mathrm{H}$, et al. High signal intensity in dentate nucleus on unenhanced T1-weighted MR images: association with linear versus macrocyclic gadolinium chelate administration. $R a$ diology 2015;275:803-09 CrossRef Medline

26. Thomsen HS. T1 hyperintensity in the brain after multiple intravenous injections of gadolinium-based contrast agents. Acta Radiol 2016;57:389-91 CrossRef Medline

27. Minagar A, Barnett MH, Benedict RH, et al. The thalamus and multiple sclerosis: modern views on pathologic, imaging, and clinical aspects. Neurology 2013;80:210-19 CrossRef Medline

28. Filippi M, Rocca MA, Barkhof F, et al; Attendees of the Correlation between Pathological MRI Findings in MS Workshop. Association between pathological and MRI findings in multiple sclerosis. Lancet Neurol 2012;11:349-60 CrossRef Medline

29. Roccatagliata L, Vuolo L, Bonzano L, et al. Multiple sclerosis: hyperintense dentate nucleus on unenhanced T1-weighted MR images is associated with the secondary progressive subtype. Radiology 2009; 251:503-10 CrossRef Medline

30. Ramalho J, Ramalho M, AlObaidy M, et al. T1 signal-intensity increase in the dentate nucleus after multiple exposures to gadodiamide: intraindividual comparison between 2 commonly used sequences. AJNR Am J Neuroradiol 2016;37:1427-31 CrossRef Medline

31. Ramalho J, Ramalho M, AlObaidy M, et al. Technical aspects of MRI signal change quantification after gadolinium-based contrast agents' administration. Magn Reson Imaging 2016;34:1355-58 CrossRef Medline

32. Rocca MA, Amato MP, Enzinger C, et al; MAGNIMS Study Group. Clinical and imaging assessment of cognitive dysfunction in multiple sclerosis. Lancet Neurol 2015;14:302-17 CrossRef Medline

33. Tröster AI, Woods SP, Fields JA. Verbal fluency declines after pallidotomy: an interaction between task and lesion laterality. Appl Neuropsychol 2003;10:69-75 CrossRef Medline

34. Mariën P, Ackermann H, Adamaszek M, et al. Consensus paper language and the cerebellum-an ongoing enigma. Cerebellum 2014;13:386-410 CrossRef Medline

35. Sbardella E, Upadhyay N, Tona F, et al. Dentate nucleus connectivity in adult patients with multiple sclerosis: functional changes at rest and correlation with clinical features. Mult Scler 2017;23:546-55 CrossRef Medline

36. Filippi M, Rocca MA, De Stefano N, et al. Magnetic resonance tech niques in multiple sclerosis: the present and the future. Arch Neurol 2011;68:1514-20 CrossRef Medline

37. Sandroff BM, Schwartz CE, DeLuca J. Measurement and maintenance of reserve in multiple sclerosis. J Neurol 2016;263:2158-69 CrossRef Medline 